\title{
Type 1 diabetes genetic susceptibility markers and their functional implications
}

\author{
Yongsoo Park* \\ Department of Internal Medicine, Hanyang University College of Medicine, Seoul, Korea
}

Type 1 diabetes (T1D) is a chronic autoimmune disease characterized by selective destruction of pancreatic $\beta$-cells resulting in insulin deficiency. The genetic determinants of T1D susceptibility have been linked to several loci, in particular to the human leukocyte antigen (HLA) region, which accounts for $50 \%$ of the genetic risk of developing T1D. Multiple genes in the HLA region, which are in strong linkage disequilibrium, are thought to be involved. Another important locus, with a smaller effect on genetic predisposition to T1D, is the insulin gene. The advent of numerous single nucleotide polymorphism markers and genome screening has enabled the identification of dozens of new T1D susceptibility loci. Some of them appear to predispose to T1D independently of the HLA and may be important in families with T1D who lack strong HLA susceptibility. Other loci may interact with each other to cause susceptibility. The autoimmune response against $\beta$-cells can also be triggered by environmental factors in the presence of a predisposing genetic background. Deciphering the environmental and genetic factors involved should help to understand the origin of T1D and aid in the design of individualized prevention programs.

Key words: Type 1 diabetes mellitus, Autoimmune diseases, Genetics, HLA antigens.

\section{Introduction}

Type 1 diabetes (T1D) is a chronic autoimmune disease characterized by destruction of the insulin-producing $\beta$-cells of the pancreas. Although the American Diabetes Association recommends an etiological classification, diabetes resulting from deficient insulin secretion, rather than deficient insulin action, is usually classified as T1D. The prevalence of T1D ranges from $<5$ in every 100,000 individuals in Asian countries to as many as 39.9 per 100,000 in European and other Western countries [1]. A distinguishing feature of T1D is the presence of anti-islet autoantibodies, including antibodies against glutamic acid decarboxylase (GAD) 65, against insulinoma-associated antigen (IA-2), against insulin, against zinc transporter 8 (ZNT8), and against islet cell autoantigen (ICA). More than 90\% of individuals with T1D test positive for at least one such autoantibody, and the presence of autoantibodies is used to identify the relatives of patients who are at increased risk of the disease [2-4].

Although genetic factors are strong determinants of the disease, more than $90 \%$ of individuals who present with newonset diabetes do not have a relative with T1D [3]. The overall risk of T1D for the siblings of T1D patients is approximately $6 \%$, which is 15 fold higher than in the general population [5]. The most important susceptibility alleles are found within the human leukocyte antigen (HLA) region, which has an odds ratio for disease of approximately 6.8 [6].

\footnotetext{
Received: 17 October 2013, Revised: 2 January 2014, Accepted: 19 February 2014, Published: 30 June 2014

*Corresponding author: Yongsoo Park, M.D., Ph.D.

Department of Internal Medicine, Hanyang University College of Medicine, 222 Wangsimni-ro, Seongdong-gu, Seoul 133-791, Korea

Telephone: +82-2-2298-2283; Fax: +82-2-2298-2283; E-mail: parkys@hanyang.ac.kr

Conflict of interest: We declare that we do not have any conflicts of interests.
}

(c) This is an open-access article distributed under the terms of the Creative Commons Attribution Non-Commercial License (http://creativecommons.org/licenses/by-nc/3.0/) which permits unrestricted non-commercial use, distribution, and reproduction in any medium, provided the original work is properly cited.

(c) Copyright 2014 by the Korean Society of Medical Genetics 


\section{Inheritance of Immunologic Markers and Development of Diabetes}

About half of monozygotic twins of T1D patients go on to develop diabetes [5]. There is a large difference between the concordance rates for T1D in monozygotic (50\%) and dizygotic $(-5 \%)$ twins, which demonstrates the importance of genetic factors in the development of T1D. However, since the rate is not $100 \%$, other environmental factors clearly play an important role.

The non-obese diabetic (NOD) mouse is an inbred strain that develops insulitis and diabetes that closely resembles human T1D. Autoimmune diabetes in NOD mice and in humans shares many genetic and pathophysiological characteristics $[7,8]$. In both species, genes within and outside the major histocompatibility complex ( $\mathrm{MHC}$, whose homolog in humans is HLA) contribute to disease susceptibility. The odds associated with the non-MHC genes are considerably smaller than with the MHC genes. A number of non-MHC T1D-associated genetic variants, out of which the insulin gene is most important, are thought to increase disease susceptibility in humans [9-11]. A comprehensive list of the susceptibility loci, derived both from the syntenic candidate genes of mouse homologs and from linkage analysis in humans can be found in Table 1, together with LOD scores from the 2005 T1D Genetics Consortium (T1DGC) scan $[12,13]$. Although as many as 18 genetic loci have been implicated in T1D susceptibility, more than $50 \%$ of the genetic risk of developing diabetes is thought to be conferred by the HLA region alone $[6,10,12,14]$.

Table 1. Genetic contributions of the known susceptibility loci derived from candidate gene and linkage analyses to the risk of type 1 diabetes

\begin{tabular}{|c|c|c|c|c|}
\hline Locus & Chromosome & Candidate genes & Markers & $\mathrm{LOD}^{\mathrm{a}}$ \\
\hline IDDM1 & $6 p 21.3$ & $H L A-D R / D Q$ & TNFA & 116.38 \\
\hline IDDM2 & $11 p 15.5$ & Insulin VNTR & D11S922 & 1.87 \\
\hline PTPN22 & $1 p 13$ & PTPN22 (Lyp) & $\mathrm{SNP}=\mathrm{R} 620 \mathrm{~W}$ & $N R$ \\
\hline IDDM3 & $15 q 26$ & & D15S107 & NR \\
\hline IDDM4 & $11 q 13.3$ & MDU1, ZFM1, RT6, ICE, LRP5, FADD, CD3 & FGF3, D11S1917 & NR \\
\hline IDDM5 & $6 q 25$ & SUMO4, MnSOD & ESR, a046Xa9, SNP=M55VA allele 163 (G) & NR \\
\hline IDDM6 & $18 q 12-q 21$ & $J K(K i d d)$, ZNF236 & D18S487, D18S64 & NR \\
\hline IDDM7 & $2 q 31-33$ & NEUROD & D2S152, D251391 & 3.34 \\
\hline IDDM8 & $6 q 25-27$ & & D6S281, D6S264, D6S446 & NR \\
\hline IDDM9 & $3 q 21-25$ & & D3S1303, D3S1589, D3S3606 & NR \\
\hline IDDM10 & 10p11-q11 & $S D F-1$ & D10S565, D10S193 & 3.21 \\
\hline IDDM11 & 14q24.3-q31 & ENSA, SEL-1L & D14S67 & NR \\
\hline IDDM12 & $2 q 33$ & CTLA-4 & (AT)n 3' UTR, A/G Exon 1 & 3.34 \\
\hline IDDM13 & $2 q 34$ & IGFBP2, IGFBP5, NEUROD, HOXD8 & D2S137, D2S164, D2S1471 & NR \\
\hline IDDM15 & $6 q 21$ & & D6S283, D6S434, D6S1580 & 22.39 \\
\hline IDDM16 & $14 q 32$ & $I G H$ & & NR \\
\hline IDDM17 & 10q25 & & D10S1750, D10S1773 & NR \\
\hline \multirow[t]{10}{*}{ IDDM18 } & $5 Q 31.1-33.1$ & $I L 12 B$ & IL12B & NR \\
\hline & $1 q 42$ & & D1S1617 & NR \\
\hline & 16p12-q11.1 & & D16S3131 & 1.88 \\
\hline & $16 q 22-q 24$ & & D16S504 & 2.64 \\
\hline & $17 q 25$ & & & NR \\
\hline & $19 q 11$ & & & NR \\
\hline & 3p13-p14 & & D3S1261 & 1.52 \\
\hline & $9 q 33-q 34$ & & D9S260 & 2.20 \\
\hline & $12 q 14-q 12$ & & D12S375 & 1.66 \\
\hline & 19p13.3-p.13.2 & & INSR & 1.92 \\
\hline
\end{tabular}

NR, not reported in the 2005 T1DGC scan [12], which applies to loci with no significant evidence for linkage in that study.

${ }^{a}$ According to the criteria of Lander and Kruglyak (1995) [13], an LOD $\geq 3.6$ is considered significant. 


\section{Major Histocompatibility Complex}

Several studies have now shown that both the HLA-DQ and the HLA-DR genes influence T1D susceptibility. It has also become evident that susceptibility as well as protective alleles can be found at the DRB1, DQA1, and DQB1 loci. Studies have shown that HLADR3 or -DR4, or both alleles, strongly increase susceptibility to T1D, whereas HLA-DR 15 has a protective effect across several populations $[6,7,9]$. In Asian populations, studies identified a positive association of T1D with $\mathrm{HLA}-\mathrm{DRB} 1^{*} 09$, and a negative association with HLA-DRB1*11(12) $[10,11,14,15]$. DQ2 (DQB1* 0201-DQA $1^{*} 0501$ ) and D08 (DQB1*0302-DQA $1^{*} 0301$ ), which occur in strong linkage disequilibrium (LD) with DR3 and DR4, respectively, are strongly associated with the disease not only in Caucasian but also in Asian populations, especially when present in DR3/DR4 heterozygotes [10-12,14-16]. In T1D, the HLADR3-D02 and -DR4 -D08 haplotypes are referred to as 'highrisk haplotypes'. HLA-DR 15 is in LD with D06.2 (DQA 1*0102$\mathrm{DOB} 1^{*} 0602$ ) and is known as 'a protective haplotype' $[11,17]$. The protection conferred by the D06.2 haplotype is assumed to be dominant, as even among autoantibody $(+)$ relatives, this haplotype protects from the development of diabetes.

In many countries, however, the incidence of T1D has been increasing in young children at a faster rate than can be explained by genetic change alone, highlighting the role of environmental factors $[9,18,19]$. There is evidence of an increasing trend over the past 20 years in the incidence of T1D, and especially in individuals with the protective HLA haplotypes, such as HLA-DQB $1{ }^{*} 0602$. These findings are alarming, and they are driving the search for environmental factors that may be responsible for triggering T1D, such as changes in exposure to infectious, environmental, or nutritional agents.

\section{HLA Molecules in a Trimolecular Complex Determine T1D Susceptibility}

In addition to the high-risk and protective variants, there are other, less common, HLA haplotypes that influence T1D susceptibility [17]. The peptide-binding selectivity of HLA class II molecules was found to be influenced by their genetic polymorphisms, and the overall risk of T1D associated with the HLA-DR and -DQ molecules is determined by a combination of alleles (haplotypes and genotypes) in any individual. Studies of peptide binding to the HLA antigen have indicated that different HLA alleles may bind different peptide epitopes from a single candidate diabetes antigen, such as GAD65 or insulin [20]. Consistent with this observation is the finding that the disease process is affected by multiple peptides. This, in turn, may mean that multiple amino-acid residues in class II molecules (i.e., alleles) are also involved. The antigen-specific pathogenic $T$ cell response is triggered either by several $\beta$ cell-derived peptides at the same time, or by a single peptide, with subsequent interand intramolecular spreading of the autoimmune response and expansion of the T cell repertoire [21] .

Some studies have reported a modulatory effect of HLA-DPB1 alleles, as well as class I molecules, on the DR/DQ-associated risk of T1D. These studies suggest that susceptibility of the DP locus is conferred primarily by the DPB $1^{*} 0301$ and DPB1 ${ }^{*} 0202$ alleles, while protection is conferred by DPB $1^{*} 0402[22,23]$.

\section{MHC Class I Chain-related Gene A}

MHC class I chain-related gene A (MICA) also resides in the human MHC locus. The trinucleotide repeat (GCT)n microsatellite polymorphisms within exon 5 code for polyalanine in the transmembrane (TM) region of the MICA molecule. In most populations, 5 MICA microsatellite alleles have been identified, consisting of 4, 5, 6, and 9 GCT repetitions, and 5 repetitions with an additional nucleotide insertion (GCT to GGCT). Accordingly, these alleles have been named $A 4, A 5, A 6, A 9$ and $A 5$.1. There are few studies describing the functional consequences of these polymorphisms. It has been suggested that the dihydrophobic Leu-Val tandem sequence at position 344-345 in the cytoplasmic tail of MICA is responsible for targeting the protein to the basolateral plasma membrane of gut epithelial cells, which is the prime site of contact with effector NK and intraepithelial T cells. In MICA-A5.1, insertion of an additional $\mathrm{G}$ creates a frame-shift mutation resulting in a premature termination codon within the TM region, as a result of which the MICA-A5.1 molecule is not translated all the way to the Leu-Val sequence at position 344-345. The LeuVal sorting signal is thus removed, which results in MICA-A5.1 molecules being localized to the apical plasma membrane instead of the basolateral membrane [24].

A number of studies have reported an association between the MICA alleles and several autoimmune disorders, such as juvenile rheumatoid arthritis, Addison's disease, psoriasis and ankylosing spondylitis. Likewise, there are reports describing an association between different MICA alleles and T1D in several populations $[25,26]$. MICA-A9 was found to be positively associated with T1D in a Chinese population, whereas in Koreans, MICA-A4 
was found to be positively associated, and MICA-A6 negatively associated with T1D.

\section{The Variable Number of Tandem Repeats (VNTR) Locus of the Insulin Gene}

Several studies have shown a strong association between T1D and the variable number of tandem repeats (VNTR) locus, found approximately 596 bases upstream of the transcription start site of the insulin gene on chromosome 11 p15.5 (Fig. 1). This locus is thought to contribute about 10\% of the variation in T1D susceptibility. Based on the number of tandem repeats of 14-15 bp G-rich sequences, the VNTR alleles have been grouped into 3 classes: class I alleles (20-63 repeats), class II alleles (64-139 repeats) and class III alleles (140-210 repeats). The short, class I alleles are generally predisposing, especially in the homozygous state, with a relative risk $>2$. Conversely, class III alleles are associated with dominant protection. Among Caucasians, class I alleles are the most common, with a frequency of nearly $80 \%$; class III alleles are less frequent (nearly 20\%), and class II are the least common variants $[9,27]$. By contrast, class I alleles occur in more than $90 \%$ of the Asian population $[10,11,14]$.

The exact mechanism by which these alleles influence the risk of T1D is not well understood. As the VNTR locus is in a non- coding region, its allelic variants may exert their influence by modulating the expression levels of insulin in the thymus and pancreas. On the one hand, increased transcription of the insulin gene in the thymus is associated with the protective, class III alleles, while, on the other, low levels of transcription are found in the presence of the predisposing, class I alleles. However, the situation is reversed in the pancreas, where higher insulin mRNA expression is associated with class I alleles [28]. These findings suggest that the negative selection of autoreactive thymocytes is dose-dependent, and that elevated levels of insulin in the thymus may promote efficient deletion of autoreactive T cells for insulin and its precursors. This, in turn, would induce central immune tolerance to this important autoantigen involved in the pathogenesis of T1D.

\section{The Cytotoxic T Lymphocyte Associated Antigen 4 Gene}

The cytotoxic T lymphocyte-associated antigen 4 (CTLA-4) gene on chromosome 2 (2q33) has been associated with many autoimmune diseases (Fig. 2) [29]. Susceptibility to T1D was found to be linked to a single nucleotide polymorphism (SNP) at the 5 ' end of CTLA-4 rather than a polymorphism in the 3'UTR [30]. A combined analysis of families from several European popula-

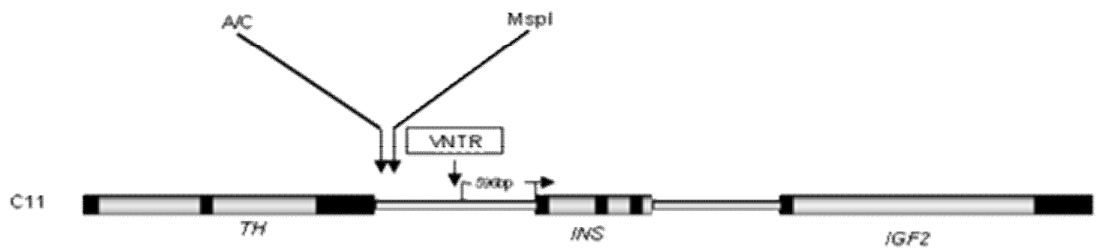

\begin{tabular}{|c|c|c|}
\hline \multicolumn{2}{|c|}{ INS-VNTR } \\
\hline $\begin{array}{c}\text { G-rich repeat units (14-15bp) in the promoter region } \\
\text { (20-63 repeats) }\end{array}$ & $\begin{array}{c}\text { Class II } \\
\text { (64-139 repeats) }\end{array}$ & $\begin{array}{c}\text { Class III } \\
(140-210 \text { repeats })\end{array}$ \\
\hline $\begin{array}{c}\text { Lesserinsulin } \\
\text { production in } \\
\text { thymus \& more } \\
\text { in pancreas }\end{array}$ & $\begin{array}{c}\text { More insulin } \\
\text { production in } \\
\text { thymus \& lesser } \\
\text { in pancreas }\end{array}$ \\
\hline \begin{tabular}{c|c|} 
Common \\
Predisposing
\end{tabular} & $\begin{array}{c}\text { Rare } \\
\text { Pery Rare } \\
\text { Not Known }\end{array}$ \\
\hline
\end{tabular}

Fig. 1. Schematic presentation of the insulin gene (INS) and variable numbers of tandem repeats (VNTR) locus, with special emphasis on the association of differential expression pattern of VNTR in thymus and pancreas with type 1 diabetes (T1D) susceptibility. VNTRs associated with T1D are located 596 bp upstream of the transcription site in the promoter region of the gene. The lower half of the figure explains the correlation between INS-VNTR alleles and insulin production that leads to T1D susceptibility or resistance. Dark-shaded boxes are exons; light-shaded boxes are introns. 
tions revealed linkage with $\mathrm{T1D}$ and autoimmune thyroid disease in the $6.1 \mathrm{~kb}$ region of CTLA-4. The $+49 \mathrm{G}-\mathrm{CT} 60 \mathrm{G}$ haplotype was identified as predisposing and the $+49 \mathrm{~A}-\mathrm{CT} 60 \mathrm{~A}$ as protective.

CTLA-4, expressed on the surface of activated T cells, binds to ligand B7, expressed on the surface of antigen presenting cells (APCs), and down-regulates the expression of the interleukin (IL)-2 receptor, which is followed by decreased synthesis of IL-2. CTLA-4 is also a molecule through which CD4+CD25+ regulatory T cells exert their suppressive activity. There are 2 known CTLA4 isoforms: the main isoform is a full-length TM glycoprotein expressed on the surface of activated T cells, and the other is a soluble form (sCTLA-4). The latter is generated by alternative splicing of the TM domain and is mainly expressed by inactivated T cells. Allelic variation at the CTLA-4 locus determines the expression level of the soluble isoform [30]. The latter may increase the inhibitory effects of CTLA- 4 by competing with CD28 for binding to costimulatory B7 molecules on the APCs. The ratio of sCTLA-4 to full-length CTLA-4 transcripts in unstimulated CD4 T cells was found to be $50 \%$ lower in individuals with a predisposing haplotype. Therefore, the level of CTLA-4, especially of the soluble form, may affect the risk of developing autoimmune diseases, including T1D.

\section{The Protein Tyrosine Phosphatase N22 Gene}

The protein tyrosine phosphatase N22 (PTPN22) gene on chromosome 1p13.2 encodes a lymphoid-specific phosphatase known as lymphoid tyrosine phosphatase (Lyp), which plays an

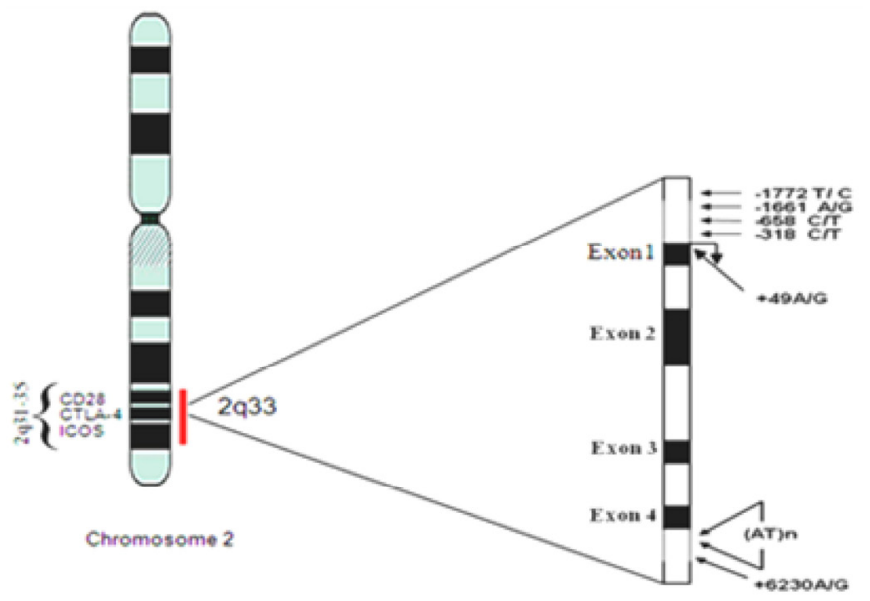

Fig. 2. Schematic presentation of the genetic structure of the cytotoxic T lymphocyte-associated antigen-4 (CTLA-4) on chromosome $2 q 33$ is shown. Various polymorphisms in the CTLA-4 gene in the 5'UTR- and 3'UTR-regulatory regions and exon 1 have been studied extensively in patients with type 1 diabetes. Dark-shaded boxes are exons; lightshaded boxes are introns. important role in the autoimmune response (Fig. 3). It dephosphorylates the Lck, Fyn, and ZAP-10 proteins, which are known to be important in T cell signaling. Lyp binds to C-terminal Src kinase (Csk) and to the adaptor molecule Grb2 (growth factor receptor-bound protein 2), resulting in down-regulation of activated T cells [31].

Recently, a positive association was found in several populations between a nonsynonymous SNP C1858T in codon 620 of the PTPN22 gene and T1D $[32,33]$. 1858T is a rare allele that changes the amino acid from arginine (R) to tryptophan (W) at position 620 , resulting in disruption of the proline-rich binding motif 'PxxPxR', which is important for Lyp binding to both Csk and Grb2. As a result, the reduced binding capacity of W620 Lyp to Csk is believed to disrupt the function of Csk as a down-regulator of T cell activation. The 1858T allele has been positively associated with other autoimmune diseases, including rheumatoid arthritis and systemic lupus erythematosus. However, several studies found that in Asian populations it was not polymorphic, and was not associated with T1D [14].

\section{Other Candidate Markers and Lessons from the} Recent Genome-wide Association Studies

During the past 3 decades many genetic studies have been devoted to the search for other T1D susceptibility loci, either by

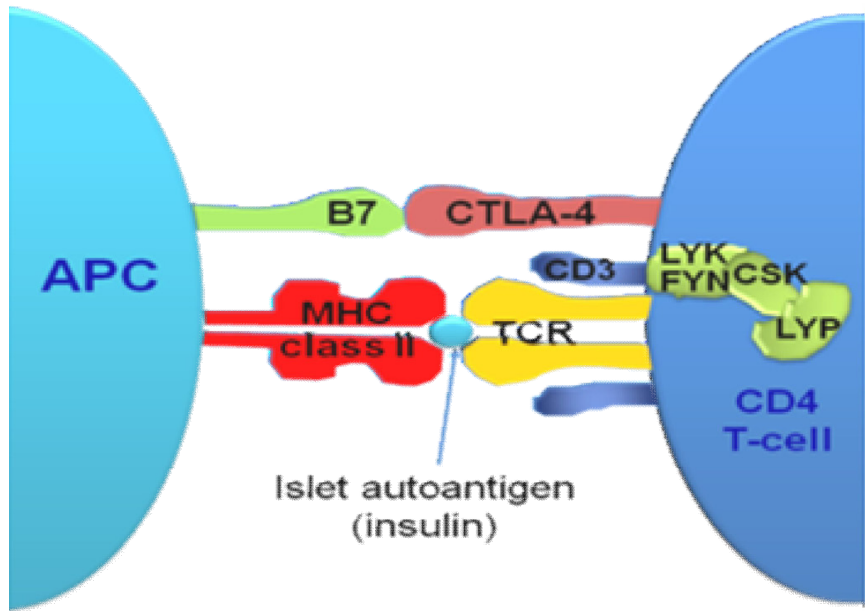

Fig. 3. Schematic presentation of the lymphoid tyrosine phosphatase (Lyp ) and its interaction with other signaling molecules are shown. An islet $\beta$-cell autoantigen (insulin peptide) is presented to CD4 T cells by the human leukocyte antigen (HLA) class II molecules on an antigen presenting cell (APC), leading to T cell activation. Like the cytotoxic T lymphocyte-associated antigen-4 (CTLA-4), LYP is an inhibitor of T cell activation and is encoded by the protein tyrosine phosphatase N22 (PTPN22) gene. The LYP-CSK (C-terminal Src kinase) complex inhibits Lck signaling after HLA-peptide-TCR engagement. MHC, major histocompatibility complex; TCR, T cell receptor. 
linkage or by association with candidate genes. Although finemapping of the intervals has not yet determined the identity of the putative susceptibility genes, important candidate genes have been identified [10-12,34,35]. A susceptibility locus close to the IL12B gene was identified and mapped to chromosome $5 q 31.1$ - q33.1. IL12B encodes the IL-12 p40 subunit, and IL12 affects the $T$ cell response, which may be important in T1D pathogenesis. LD is confined to a $30 \mathrm{~kb}$ region carrying IL12B, the only known gene in the region. Several polymorphisms have been identified in IL12B, with the A allele of C1159A showing strong LD with T1D susceptibility [35]. Fine-mapping in T1D patients have also narrowed the relevant region on chromosome 10q15, applying a multilocus test using tag SNPs [36]. The biological candidate gene IL2RA (CD25) in that region, which encodes a subunit (IL-2R $\alpha$ ) of the high-affinity IL-2 receptor complex was found to be associated with T1D. This study indicated the utility of tag SNPs in a chromosomeregional test of disease association and justify future fine mapping of the causal variant in the region. Moreover, the T1DGC has also assembled and genotyped a large collection of multiplex families. By utilizing genome-wide linkage scan data and familybased association methods, they confirmed previously reported T1D associations at the insulin gene [27], a region on 2q24 near interferon induced with helicase C domain 1 (IFIH1) [37], and a region on $16 p 13$ near $\mathrm{C}$-type lectin domain family 16 , member A (CLEC16A/KIAA0350) [38] and identified an additional disease association on chromosome 21q22.3 in the ubiquitin associated and $\mathrm{SH} 3$ domain-containing protein $\mathrm{A}$ (UBASH3A) locus [39].

Although there is a large genetic component to T1D susceptibility, uncovering the non-HLA genes contributing to T1D has proven difficult. It was not the advent of genome-wide association studies (GWAS), until only 5 loci (the insulin, the PTPN22, the CTLA-4, the IL2RA, and the KIAA0350) had been fully established to be associated with T1D [39]. The majority of the other reported associations in the pre-GWAS era $[40,41]$ were found to be doubtful, where an initial report of association could not be replicated in subsequent studies by other investigators. Inconsistent findings might be attributed to small sample sizes. The candidate gene approach (where genomic regions were studied based on biological reasoning) and family-based linkage methodologies, which are limited in terms of hypothesis and power are no longer popular and can be replaced by the recent unbiased GWAS. They allow investigators to scan the genome for variants conferring risk for disease without prior hypotheses. They have also leveraged large, well-powered sample sizes combined with sound statistical analyses.
Such GWAS have revealed multiple regions of the genome to be consistently associated with T1D [42,43]. More recent reports have been a consequence of combining of multiple datasets from independent investigators in meta-analyses, which have more power to pick up additional variants contributing to T1D susceptibility. In addition to the repertoire of over 40 loci already demonstrated to be associated, in a meta-analysis of T1D GWAS, which combines 6 large studies, 3 new signals residing at the chromosomal locations 13q22, 2p23, and 6q27 were found to be replicated in independent samples [44]. Further work will be required to fully validate the role of these loci in the pathogenesis of T1D. These latest associated regions may add to the growing repertoire of gene networks predisposing to T1D.

\section{Genetic susceptibility to T1D in Koreans}

Although all T1D cases have common traits and selective destruction of pancreatic $\beta$-cells is always observed, the clinical and immunologic characteristics of T1D differ considerably between Asian and Caucasian populations [1]. While reporting a low incidence of T1D in Asia, researchers were unclear about the prevalence of islet-specific autoantibodies in Asian T1D patients $[1,4,14]$. A subsequent study, using a radioligand binding assay for GAD and IA-2 in young ( $<15$ years at diagnosis) Korean T1D patients, found that the prevalences of IA-2, GAD65, and ICA autoantibodies in patients with recent-onset disease (diagnosed $<1$ year before) were comparable to those reported for Caucasians $[4,14]$.

T1D is much less frequent in Asian countries than in countries with predominantly Caucasian populations, which might suggest that Asians are genetically protected against T1D. However, the $\lambda$ s value, indicating familial clustering, was found to be high despite the low prevalence [45]. It could, therefore, be argued that if a genetic factor is responsible for the high $\lambda$ s when general preva lence is low, then susceptibility genes (whose frequencies are very low in the general population) may be segregating in T1D families.

T1D in Koreans is thought to be strongly associated with specific HLA alleles, namely DR and DQ [15]. A case-control study evaluating the DRB1-DQB1 haplotypes found that there was a difference of over 200-fold in the risk of T1D between the least susceptible and the most susceptible haplotypes. Although the risk associated with individual alleles seems to vary between populations, when comparing identical DRB1-DQB1 haplotypes the association and transmission to diabetic offspring was 
similar for Koreans and Caucasians, suggesting that these haplotypes transcend ethnic boundaries [17].

Importantly, having autoantibodies does not necessarily mean being diabetic. As expected, concordant sibs or T1D patients and multiple autoantibody (+) sibs appeared to share the same $2 \mathrm{HLA}$ DR-D0 haplotypes. However, a typical 1:2:1 haplotype sharing was observed between patients with T1D and single antibody (+) sibs [14].

The contribution of the DQ molecule to overall disease susceptibility is thought to be genotype dependent [16]. Although the prevalence of the HLA-DR4-DOB 1*0302 haplotype did not differ between T1D patients and controls, the HLA-DR3/4-DQB1*0302 genotype was linked to an increased risk, indicating a synergistic effect. This synergism may be explained by the finding that the transmission of $\mathrm{DQB} 1{ }^{*} 0302$ varied depending on whether the homologous haplotype was DR3 or not; DQB 1*0302 was more frequently transmitted to the affected sibling when there was DR3 on the other chromosome. Thus, a significant association of $\mathrm{DOB} 1{ }^{*} 0302$ with the DR3/4 genotype must exist. The situation was the reverse for the DOB $1^{*} 0401$ haplotype, which is also positively associated with T1D; DQB1*0401 was not frequently transmitted to diabetic offspring in the presence of DR3, suggesting a significant association of DQB $1{ }^{*} 0401$ with DR4/X.

The frequencies of T1D-associated HLA antigens correlate well with T1D incidence rates worldwide $[14,46]$. Generally, the DR3 and DR4 haplotypes are common in Caucasians, which means that the prevalence of T1D is likely to be higher due to easy formation of the D0 0201/0302 dimer. Conversely, the frequency of the DRB1 genotypes among Korean T1D patients points to a much lower contribution of DR3 and DR4, especially the DR3/4 genotype. In Koreans, it appears, moderate-risk haplotypes are more frequent whereas susceptible haplotypes are rare. When determining population-specific susceptibility, rare genotypes are important: among the DR genotypes, that includes DR3/9, DR9/9, and DR3/X (X: other than 3,4), none of which are susceptible in Caucasians. As the contribution of HLA-DR3/4 is relatively minor in Korea, it may be just the tip of an iceberg, with many more minor genes (including non-HLA) and less frequent genotypes at play. Moreover, when we assessed the association of the different DR4 subtypes with susceptible D08 and neutral DQ4 (DQA 1*0301-DQB1*0401) haplotypes in Korean general population, D08 is usually combined with protective DR4 subtypes (DRB $1{ }^{*} 0403,{ }^{*} 0406$ ), while D04 is in LD with susceptible DRB1*0405. High-risk DR4 subtypes (DRB1 ${ }^{*} 0401$, ${ }^{*} 0402$, ${ }^{*} 0405$ ) were predominant in DR4/X, whereas protective DR4 subtypes (DRB1*0403, $\left.{ }^{*} 0406\right)$ were observed mainly in the
DR3/4 genotype [46]. In this sense, the low incidence rate in the Korean population may be explained by the counterbalancing effect of the DRB1 and DQB1 alleles in the control population. A different genetic susceptibility including a low frequency of high-risk HLA alleles could explain the lower prevalence of isletspecific autoantibodies and the low incidence of T1D, or different genetic and environmental interactions might be involved in the etiology of T1D.

\section{Clinical Heterogeneity of Diabetes in Young Asian Patients}

In Asia, diabetes among young patients has complex causation. The relative proportion of type 2 diabetes (T2D) among all diabetic cases is quite high. There is also a variable incidence of non-autoimmune types of T1D associated with insulin deficiency (T1b) , such as fulminant T1D (F1D) and virus-induced T1D. Imagawa et al. [47] reported a high prevalence (up to 20\%) of FT1D among cases of acute onset T1D in Japan, but little is known about the situation in other countries. Our own data suggest that, although T1a is the major type of childhood diabetes, FT1D exists as a hyperacute subtype of T1D that affects older children, without there being any evidence of autoimmunity. Our patients showed a complete loss of $\beta$-cell secretory capacity without evidence of recovery, necessitating long-term treatment with insulin [48]. In this context, early diagnosis and classification of patients with acute-onset diabetes may be crucial in order to minimize the formidable complications.

Latent autoimmune diabetes in adults (LADA) is a form of autoimmune-mediated diabetes, usually diagnosed based on GAD autoantibody positivity. Although several epidemiological surveys of LADA have been conducted in Caucasian and Asian populations, their reported incidence and prevalence rates vary due to the use of different diagnostic criteria. Applying comparable criteria and a similar level of ascertainment, a cross-sectional analysis was performed on 193 LADA patients diagnosed in Italy and 39 LADA patients in Korea. Using GAD autoantibody positivity as the diagnostic criterion, the prevalence of LADA was identical in Italy and in Korea (4.4\%) [49]. For Korea, this was considerably higher than the previously reported prevalence of 1.7\% in a population-based T2D survey [1]. However, as the GAD antibodies were mostly low-affinity and low-titer, this higher prevalence may not necessarily lead to a significant increase in subsequent insulin use. Furthermore, the prevalence of many other autoantibodies was also low in this population. After 
36 months of follow-up, only 3 of the 39 patients initially diagnosed with LADA had become insulin-dependent, and they were all positive for multiple autoantibodies (GAD, IA-2 and ZnT8 antibody) [49]. Importantly, this demonstrates that true insulin dependency, in the beginning indicated by multiple antibody positivity, has not increased in the Korean population.

\section{Conclusion}

T1D is a complex, polygenic, organ-specific autoimmune disease, with both genetic and environmental factors contributing to its development. At present, HLA genes, including the HLAD0 and -DR haplotypes, are considered to be its strongest risk factors. The non-HLA genetic susceptibility is influenced by multiple genes and, under certain conditions, may modify the risk contributed by the HLA genes. Fine mapping and functional studies are revealing the complex mechanisms whereby immune tolerance is lost, involving multiple aspects of adaptive immunity. The triggering of these events by dysregulation of the innate immunity has also been suggested by genetic evidences. At this stage, pathway-directed or system biologyaided functional studies are necessary to understand their exact influence on overall disease susceptibility and/or protection. Research also needs to focus on the autoantigenic peptides that are of critical importance for diabetes, their binding affinity with particular MHCs, and the type and specificity of the T cells involved in the immunological cascade that leads to damage to the pancreatic $\beta$ cells (especially via the interaction with candidate antigens). Other contributors to disease susceptibility that may warrant further study include differential expression of candidate antigens and their variants in the thymus, as well as non-germline genes and environmental factors [50].

In addition to the HLA locus, at least 40 other loci are now known to influence susceptibility to T1D. Some of these appear to predispose individuals to T1D independently of HLA, and they may play an important role in families who lack strong HLA susceptibility. The effect of non-HLA genes on T1D susceptibility appears to be modest. However, studying their involvement in various population groups may provide a vital clue to the pathways involved in the development of T1D, and lead to effective methods of intervention. GWAS revealed genomic risk loci that potentially have an impact on disease and phenotypic traits. This extensive resource will guide us to novel directions for personalized medicine, including disease risk prediction, prevention and intervention. To make the initial identification of an association contribute to precision treatment of patients, the integrative analysis of system-wide data on genome variation, gene expression, transcription factor binding, epigenetic profiles and chromatin conformation is crucial in understanding the biological mechanism that underlie the association. The generation of these data mainly relies on next-generation sequencing. Mass spectrometry-based proteomics now offers additional possibilities for the identification of functional genome variants and, in particular, for the identification and characterization of (differentially) bound protein complexes as well as physiological target genes. Such data will also be crucial in developing personalized molecular medicine solutions based on individuals' genetic make-up.

\section{Acknowledgements}

This research was supported by the Basic Science Research Program of the National Research Foundation of Korea (NRF) funded by the Ministry of Education, Science and Technology (2010-0010898) and the Korea Health 21 R\&tD Project, Ministry of Health and Welfare, Republic of Korea (A102065).

\section{References}

1. Park Y, Lee $H_{1}$ Koh CS, Min H, Rowley M, Mackay IR, et al. The low prevalence of immunogenetic markers in Korean adult-onset IDDM patients. Diabetes Care 1996;19:241-5

2. Gianani R, Eisenbarth GS. The stages of type 1 A diabetes: 2005. Immunol Rev 2005;204:232-49.

3. Park Y, Eisenbarth GS. Aetiology and epidemiology of type 1 diabetes. Topic Endocrinol 1998;10:2-6.

4. Park Y, Lee $H_{1}$ Takino $H_{1}$ Abiru N, Kawasaki E, Eisenbarth GS. Evaulation of the efficacy of the combination of multiple autoantibodies to isletspecific antigens in Korean type 1 diabetic patients. Acta Diabetol 2001; 38:51-6.

5. Redondo MJ, Jeffrey J, Fain PR, Eisenbarth GS, Orban T. Concordance for islet autoimmunity among monozygotic twins. N Engl J Med 2008;359: 2849-50

6. Concannon P, Rich SS, Nepom GT. Genetics of type 1A diabetes. N Engl J Med 2009;360:1646-54.

7. Eisenbarth GS. Type I diabetes mellitus. A chronic autoimmune disease. N Engl J Med 1986;314:1360-8.

8. Ikegami H, Fujisawa T, Makino S, Ogihara T. Congenic mapping and candidate sequencing of susceptibility genes for Type 1 diabetes in the NOD mouse. Ann N Y Acad Sci 2003;1005:196-204. 
9. Steck AK, Rewers MJ. Genetics of type 1 diabetes. Clin Chem 2011;57: 176-85.

10. She JX, Marron MP. Genetic susceptibility factors in type 1 diabetes: linkage disequilibrium and functional analyses. Curr Opin Immunol 1998;10:682-9.

11. Park Y, Eisenbarth GS. Genetic susceptibility factors of Type 1 diabetes in Asians. Diabetes Metab Res Rev 2001;17:2-11.

12. Concannon P, Erlich HA, Julier C, Morahan G, Nerup J, Pociot F, et al. Type 1 diabetes: evidence for susceptibility loci from four genome-wide linkage scans in 1,435 multiplex families. Diabetes 2005;54:2995-3001.

13. Lander E, Kruglyak L. Genetic dissection of complex traits: guidelines for interpreting and reporting linkage results. Nat Genet 1995;11:241-7.

14. Park Y. Functional evaluation of the type 1 diabetes (T1D) susceptibility candidate genes. Diabetes Res Clin Pract 2007;77(Suppl 1):S110-5.

15. Park Y, Wang CY, Ko KW, Yang SW, Park M, Yang MC, et al. Combinations of HLA DR and DO molecules determine the susceptibility to insulindependent diabetes mellitus in Koreans. Hum Immunol 1998;59:794801.

16. Park Y, She JX, Noble JA, Erlich HA, Eisenbarth GS. Transracial evidence for the influence of the homologous HLA DR-DQ haplotype on transmission of HLA DR4 haplotypes to diabetic children. Tissue Antigens 2001;57:18591.

17. Park Y, She JX, Wang CY, Lee H, Babu S, Erlich HA, et al. Common susceptibility and transmission pattern of human leukocyte antigen DRB1-DQB1 haplotypes to Korean and Caucasian patients with type 1 diabetes. J Clin Endocrinol Metab 2000;85:4538-42.

18. Patterson CC, Dahlquist GG, Gyürüs E, Green A, Soltész G; EURODIAB Study Group. Incidence trends for childhood type 1 diabetes in Europe during 1989-2003 and predicted new cases 2005-20: a multicentre prospective registration study. Lancet 2009;373:2027-33.

19. Karvonen M, Viik-Kajander M, Moltchanova E, Libman I, LaPorte R, Tuomilehto J. Incidence of childhood type 1 diabetes worldwide. Diabetes Mondiale (DiaMond) Project Group. Diabetes Care 2000;23:1516-26.

20. Fernando $M M$, Stevens $C R$, Walsh EC, De Jager $P L$, Goyette $P$, Plenge $\mathrm{RM}$, et al. Defining the role of the $\mathrm{MHC}$ in autoimmunity: a review and pooled analysis. PLoS Genet 2008;4:e1000024.

21. Lieberman SM, Evans AM, Han B, Takaki T, Vinnitakaya Y, Caldwell JA, et al. Identification of the beta cell antigen targeted by a prevalent population of pathogenic CD8+ T cells in autoimmune diabetes. Proc Natl Acad Sci U S A 2003;100:8384-8.

22. Noble JA, Martin A, Valdes AM, Lane JA, Galgani A, Petrone A, et al. Type 1 diabetes risk for human leukocyte antigen (HLA)-DR3 haplotypes depends on genotypic context: association of DPB1 and HLA class I loci among DR3- and DR4-matched Italian patients and controls. Hum Immunol 2008;69:291-300.

23. Howson JM, Walker NM, Clayton D, Todd JA; Type 1 Diabetes Genetics Consortium. Confirmation of HLA class II independent type 1 diabetes associations in the major histocompatibility complex including HLA-B and HLA-A. Diabetes Obes Metab 2009;11(Suppl 1):31-45.
24. Suemizu H, Radosavljevic M, Kimura M, Sadahiro S, Yoshimura S, Bahram S, et al. A basolateral sorting motif in the MICA cytoplasmic tail. Proc Natl Acad Sci U SA 2002;99:2971-6.

25. Park Y, Lee $H$, Sanjeevi CB, Eisenbarth GS. MICA polymorphism is associated with type 1 diabetes in the Korean population. Diabetes Care 2001; 24:33-8.

26. Zake LN, Ghaderi M, Park Y, Babu S, Eisenbarth G, Sanjeevi CB. MHC class I chain-related gene alleles 5 and 5.1 are transmitted more frequently to type 1 diabetes offspring in HBDI families. Ann N Y Acad Sci 2002;958:30911.

27. Lucassen AM, Julier C, Beressi JP, Boitard C, Froguel $P$, Lathrop $M$, et al. Susceptibility to insulin dependent diabetes mellitus maps to a $4.1 \mathrm{~kb}$ segment of DNA spanning the insulin gene and associated VNTR. Nat Genet 1993;4:305-10.

28. Pugliese A, Zeller M, Fernandez A Jr, Zalcberg $\sqcup$, Bartlett RJ, Ricordi $C_{1}$ et al. The insulin gene is transcribed in the human thymus and transcription levels correlated with allelic variation at the INSVNTR-IDDM2 susceptibility locus for type 1 diabetes. Nat Genet 1997;15:293-7.

29. Ueda H, Howson JM, Esposito L, Heward J, Snook H, Chamberlain G, et al. Association of the T-cell regulatory gene CTLA4 with susceptibility to autoimmune disease. Nature 2003;423:506-11.

30. Ikegami H, Awata T, Kawasaki E, Kobayashi T, Maruyama T, Nakanishi $K$, et al. The association of CTLA4 polymorphism with type 1 diabetes is concentrated in patients complicated with autoimmune thyroid disease: a multicenter collaborative study in Japan. J Clin Endocrinol Metab 2006; 91:1087-92.

31. Hill RJ, Zozulya S, Lu YL, Ward K, Gishizky M, Jallal B. The lymphoid protein tyrosine phosphatase Lyp interacts with the adaptor molecule Grb2 and functions as a negative regulator of T-cell activation. Exp Hematol 2002 Mar;30(3):237-44.

32. Onengut-Gumuscu S, Ewens KG, Spielman RS, Concannon P. A functional polymorphism (1858C/T) in the PTPN22 gene is linked and associated with type I diabetes in multiplex families. Genes Immun 2004;5: 678-80.

33. Bottini N, Musumeci L, Alonso A, Rahmouni S, Nika K, Rostamkhani $M$, et al. A functional variant of lymphoid tyrosine phosphatase is associated with type I diabetes. Nat Genet 2004;36:337-8.

34. Nerup J, Pociot F; European Consortium for IDDM Studies. A genomewide scan for type 1-diabetes susceptibility in Scandinavian families: identification of new loci with evidence of interactions. Am J Hum Genet 2001; 69:1301-13.

35. Morahan G, Huang D, Ymer SI, Cancilla MR, Stephen K, Dabadghao P, et al. Linkage disequilibrium of a type 1 diabetes susceptibility locus with a regulatory IL12B allele. Nat Genet 2001;27:218-21.

36. Vella A, Cooper JD, Lowe CE, Walker N, Nutland S, Widmer B, et al. Localization of a type 1 diabetes locus in the IL2RA/CD25 region by use of tag single-nucleotide polymorphisms. Am J Hum Genet 2005;76:773-9.

37. Todd JA, Walker NM, Cooper JD, Smyth DJ, Downes K, Plagnol V, et al. Robust associations of four new chromosome regions from genomewide analyses of type 1 diabetes. Nat Genet 2007;39:857-64. 
38. Hakonarson H, Grant SF, Bradfield JP, Marchand L, Kim CE, Glessner JT, et al. A genome-wide association study identifies KIAA0350 as a type 1 diabetes gene. Nature 2007;448:591-4.

39. Concannon P, Onengut-Gumuscu S, Todd JA, Smyth DJ, Pociot F, Bergholdt $\mathrm{R}$, et al; Type 1 Diabetes Genetics Consortium. A human type 1 diabetes susceptibility locus maps to chromosome 21q22.3. Diabetes 2008;57: 2858-61.

40. Mirel DB, Valdes AM, Lazzeroni LC, Reynolds RL, Erlich HA, Noble JA. Association of IL4R haplotypes with type 1 diabetes. Diabetes 2002;51: 3336-41.

41. Biason-Lauber A, Boehm B, Lang-Muritano M, Gauthier BR, Brun T, Wollheim $\mathrm{CB}$, et al. Association of childhood type 1 diabetes mellitus with a variant of PAX4: possible link to beta cell regenerative capacity. Diabetologia 2005;48:900-5.

42. Barrett JC, Clayton DG, Concannon P, Akolkar B, Cooper JD, Erlich HA, et al; Type 1 Diabetes Genetics Consortium. Genome-wide association study and meta-analysis find that over 40 loci affect risk of type 1 diabetes. Nat Genet 2009;41:703-7.

43. Cooper JD, Walker NM, Smyth DJ, Downes K, Healy BC, Todd JA; Type I Diabetes Genetics Consortium. Follow-up of 1715 SNPs from the Wellcome Trust Case Control Consortium genome-wide association study in type I diabetes families. Genes Immun 2009;10(Suppl 1):S85-94.
44. Bradfield JP, Qu HQ, Wang K, Zhang H, Sleiman PM, Kim CE, et al. A genome-wide meta-analysis of six type 1 diabetes cohorts identifies multiple associated loci. PLoS Genet 2011;7:e1002293.

45. Ikegami H, Ogihara T. Genetics of insulin-dependent diabetes mellitus. Endocr J 1996;43:605-13.

46. Park Y. Why is type 1 diabetes uncommon in Asia? Ann NY Acad Sci 2006:1079:31-40.

47. Imagawa A, Hanafusa T, Miyagawa J, Matsuzawa Y. A novel subtype of type 1 diabetes mellitus characterized by a rapid onset and an absence of diabetes-related antibodies. Osaka IDDM Study Group. N Engl J Med 2000;342:301-7.

48. Cho YM, Kim JT, Ko KS, Koo BK, Yang SW, Park MH, et al. Fulminant type 1 diabetes in Korea: high prevalence among patients with adult-onset type 1 diabetes. Diabetologia 2007;50:2276-9.

49. Park Y, Hong S, Park L, Woo J, Baik S, Nam M, et al; KNDP Collaboratory Group. LADA prevalence estimation and insulin dependency during follow-up. Diabetes Metab Res Rev 2011;27:975-9.

50. Diez J, Park Y, Zeller M, Brown D, Garza D, Ricordi C, et al. Differential splicing of the IA-2 mRNA in pancreas and lymphoid organs as a permissive genetic mechanism for autoimmunity against the IA-2 type 1 diabetes autoantigen. Diabetes 2001;50:895-900. 